\title{
desenvolvimento $\mathrm{Aem}_{\mathrm{em}}$
}

\section{LA PERCEPCIÓN DE LA FALTA DE AGUA EN LAS COMUNIDADES WAYUU EN LA GUAJIRA (COLOMBIA). Una Propuesta para el uso de las Redes Sociales como Herramienta de Análisis de Desarrollo}

\author{
http://dx.doi.org/10.21527/2237-6453.2021.57.12107
}

Recebido em: 2/3/2021

Aceito em: 7/7/2021

\section{Katherin Pérez Mendoza ${ }^{1}$, Carlos Busón Buesa ${ }^{2}$}

\begin{abstract}
RESUMEN
El objetivo de este trabajo es analizar las posibilidades de utilizar las redes sociales como herramienta para estudiar la percepción de eventos o noticias utilizando algoritmos de análisis semánticos de discurso. Para ello realizamos una investigación sobre los discursos de comentarios en Facebook en una noticia sobre la comunidad Wayuu de forma que, pretendíamos obtener el grado y la respuesta ante una acción concreta de los usuarios de la red social, en este caso la ayuda humanitaria en una comunidad wayuu de la Península de La Guajira, Colombia que sufre las terribles consecuencias de una pertinaz sequía que agrava la situación de desequilibrio social de esta población. Pretendíamos mediante este trabajo medir la percepción de una muestra en las redes sociales frente a dicha noticia. Los estudios sobre los comentarios nos dicen mucho sobre el pensamiento colectivo de un grupo social y nos revelan pautas de respuesta frente a una publicación concreta. Utilizamos la herramienta IRaMuTeQ con base en R, que nos permite hacer uso de los comentarios de las redes sociales como una importante fuente de información para investigaciones en diversas áreas del conocimiento.
\end{abstract}

Palabras clave: Agua; redes sociales; La Guajira; Facebook; IRaMuTeQ; desarrollo sostenible.

\section{A PERCEPTION OF THE LACK OF WATER IN LA GUAJIRA IN WAYUU COMMUNITIES (COLOMBIA). THROUGH SOCIAL NETWORKS AS A MEANS OF ANALYSIS OF DEVELOPMENT}

\section{ABSTRACT}

The objective of this work is to analyze the possibilities of using social networks as a tool to study the perception of events or news using semantic discourse analysis algorithms. For this, we carried out the analysis of the discourses of the comments on Facebook about a news item about the Wayuu community so that we wanted to obtain the degree and the response to a specific action. In this case humanitarian aid. The Wayuu community of the La Guajira Peninsula, Colombia. It suffers the terrible consequences of a persistent drought that aggravates the situation of social imbalance of this population. Through this work, we wanted to measure the perception of a sample on social networks regarding said news. Studies on comments tell us a lot about the collective thought of a social group, they reveal their response patterns to a specific publication. We use the IRaMuTeQ tool. This enabled us to develop various statistical analyzes with which we could have a fairly detailed view of the response of the population to a specific event on social networks. Such a tool can undoubtedly make use of comments from social networks as an important source of materials for research in various areas of knowledge.

Keywords: Water; drought; La Guajira; facebook; IRaMuTeQ; sustainable development.

\footnotetext{
${ }^{1}$ Autora correspondente. Universidad de la Guajira - Colombia. https://scienti.minciencias.gov.co/cvlac/visualizador/generarCurriculoCv.do?cod_rh=0000055511. http://orcid.org/0000-0001-6574-1959. katherin.perez11@gmail.com

2 Universidade Estadual de Mato Grosso do Sul(UEMS) e FUNDECT/CNPq, Brasil.
} 


\section{INTRODUCCIÓN}

La península de La Guajira (en wayuunaiki³: Wajiira) se encuentra en el extremo noroeste de América Latina, pertenece a la República de Colombia limitando con el mar Caribe al norte y este, y sureste con Venezuela. La enorme variedad geográfica del territorio lo condiciona, al sudoeste podemos encontrarnos los altos picos de la Sierra Nevada de Santa Marta donde se alcanzan altitudes de más de 5.000 metros y en el noroeste, nos podemos encontrar con áreas extremadamente áridas. Este territorio lleno de contrastes tiene una población muy diversa en pueblos originarios que ocupan el territorio, como los Wayúu, Koguis, Arhuacos, Kankuamos y Wiwas. Los Wayuu son los que nos ocupan en este estudio.

El departamento de La Guajira tiene una importante población indígena, siendo que está compuesta en un $48 \%$ por comunidades indígenas de la etnia wayuu que habitan mayoritariamente en el centro y norte del departamento donde se encuentra un territorio extremamente árido, donde la escasez de agua y alimentos son la pauta. La falta de agua, provocado por su localización geográfica y actualmente agravado con la crisis climática, tal como indican los estudios del IPCC, así como el último informe ${ }^{4}$, es un problema constante que cada vez empeora, llegándose a producir severos problemas de subnutrición infantil y mortalidad por falta de agua potable y alimentos. La situación se agrava, ya que, además, no existen fuentes de rentas estables, la población vive mayoritariamente de una extensa informalidad, según los datos del Dane (2019) en algunos municipios supera el $90 \%$, lo que mantiene importantes desequilibrios sociales y económicos.

Este empobrecimiento crónico y endémico del territorio pese a ser extremadamente privilegiado en recursos naturales, tales como carbón, gas y minerales, resulta contradictorio. La falta de agua hace que sus comunidades de pueblos originarios se vean sometidos a unas condiciones de miseria extrema, para hacernos una idea de la situación, un $80 \%$ vive en la pobreza más extrema (MENDOZA, 2020), nada compatible con un territorio tan rico.

\section{PROBLEMAS SOCIOECONÓMICOS PROVOCADOS POR LA FALTA DE AGUA EN LA GUAJIRA}

El agua es un recurso fundamental para la supervivencia y el desarrollo de cualquier comunidad humana. Dependemos de ella para vivir, la vida es inviable en su ausencia. Todos los pueblos y civilizaciones que colapsaron en el pasado, en buena medida, fueron provocados por la mala gestión de este recurso tan valioso.

El problema de una adecuada gestión del agua no es un problema exclusivo de Colombia o tan siquiera de la Guajira, este es un problema extensamente sufrido en diversas partes del mundo, afectando a diversos colectivos, sobre todo las mujeres. En el trabajo Façanha (2019) se

\footnotetext{
${ }^{3}$ El wayuunaiki es una lengua de origen amerindia que es utilizado por el pueblo wayuu en la península de La Guajira, en el departamento de La Guajira, Colombia

${ }^{4}$ El cambio climático y la tierra, Informe especial del IPCC sobre el cambio climático, la desertificación, la degradación de las tierras, la gestión sostenible de las tierras, la seguridad alimentaria y los flujos de gases de efecto invernadero en los ecosistemas terrestres. Resumen para responsables de políticas. Grupo Intergubernamental de Expertos sobre el Cambio Climático, IPCC 2020. Consultado el 30 de noviembre 2020. https://www.ipcc.ch/site/assets/uploads/ sites/4/2020/06/SRCCL_SPM_es.pdf
} 
nos muestra las dificultades, sobre todo para las mujeres, en la región semiárida brasileña. Las mujeres, en Brasil, Colombia como en diversas partes del mundo son quizás, las más vulnerables y afectadas por dicho problema, ya que, son mayoritariamente las principales responsables del cuidado de las familias.

Para Reis e Carneiro (2021) la pandemia del Covid-19 refuerza las indicaciones de los Objetivos de Desarrollo Sostenible (ODS) para que los países provean las condiciones necesarias para una accesibilidad, sobre todo, para aquellos usuarios de baja renda, que suelen ser los más afectados por la carencia de servicios de agua y saneamiento básico, requeridos por la Organización de las Naciones Unidas (ONU), siendo estos un derecho humano esencial tanto para la protección de la salud como de la propia vida (p. 124).

Figura 1 - Tasa de mortalidad infantil en menores de 5 años para el periodo (2006 a 2017)

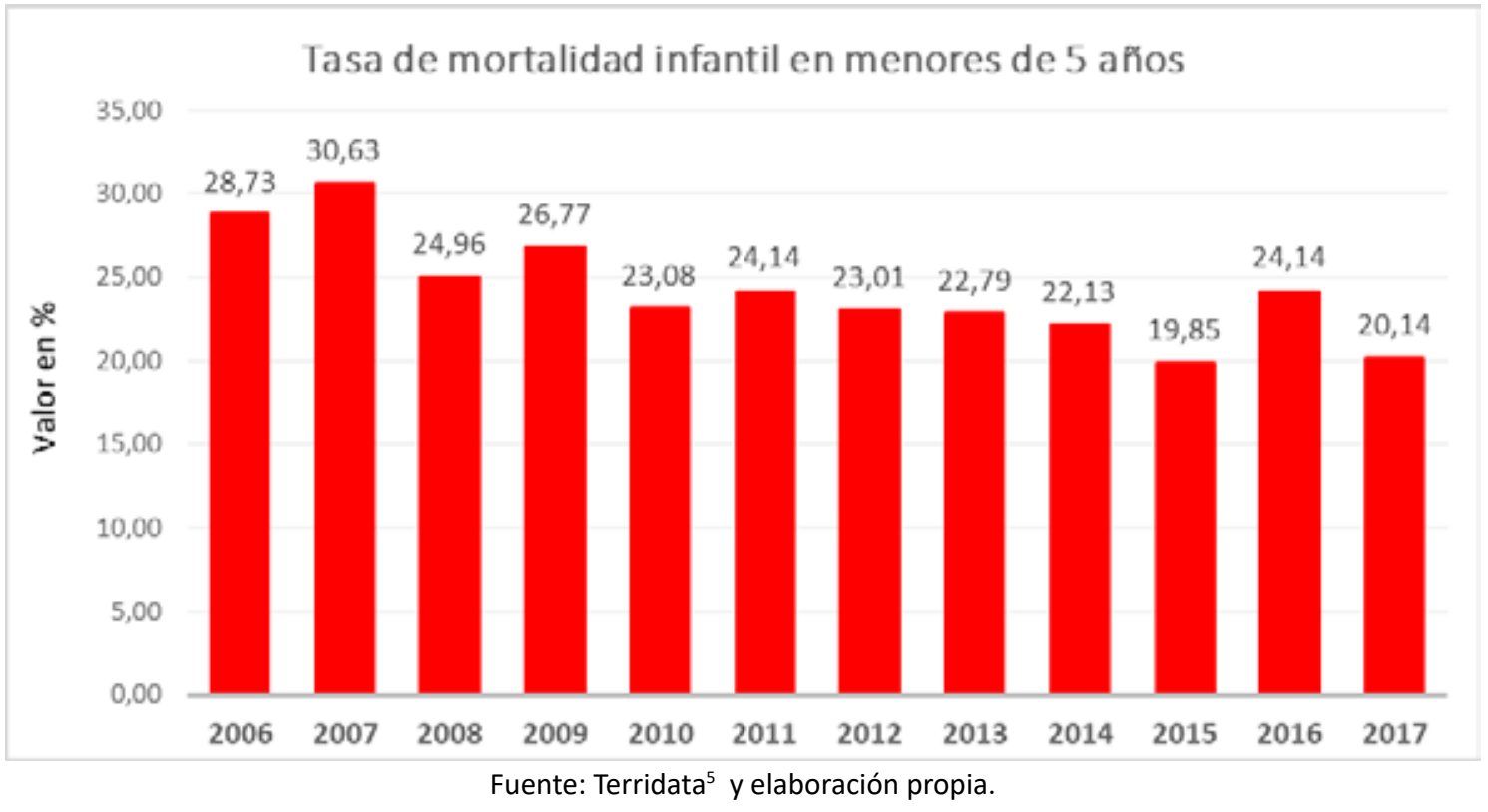

De acuerdo a los datos de la figura 1 de la tasa de mortalidad infantil en menores de 5 años de 2006 a 2017, podemos apreciar que las cifras altas, en este periodo de tiempo apenas han bajado al $20 \%$. Con esto se observa claramente que los niños están expuestos a unas condiciones precarias que condicionan su supervivencia.

Diversos estudios sobre la malnutrición infantil en La Guajira fueron realizados para analizar la realidad local, como los de Osorio et al. (2016), Morón e De Castro (2017), Bonet-Morón, Hahn-de-Castro e Hahn-de-Castro (2017) e Russell et al. (2020). Muestran que la situación es permanente por la difícil situación de las poblaciones locales ante la escasez de agua.

La vulnerabilidad local frente a las sequias es crónica. Autores como León Linares e Acosta Arias (2015), Daza Calderón e Corredor Mora (2016) e Daza-Daza, Rodríguez-Valencia e Carabalí-Angola (2018) nos muestran que pese a disponer de importantes recursos naturales (BAYONA VELÁSQUEZ, 2016), gas (JABBA, 2011) y energías alternativas (SILVA ORTEGA.; OJEDA

\footnotetext{
${ }_{5}^{5}$ Disponível em: https://terridata.dnp.gov.co/
} 
CAMARGO; CANDELO (2017) y demuestran que la desigualdad es extremadamente elevada. Esta situación resulta inaceptable en pleno siglo 21.

Como afirma Meisel-Roca (2007), en La Guajira existe un mito de las regalías redentoras, regalías que nunca llegan, a quien verdaderamente lo necesita. Este recurso no se traduce en desarrollo económico y social para la población en el territorio. Esta región ha sufrido desde los tiempos de la colonización ser sistemáticamente expoliada sin dejar, en esa época, las regalías correspondientes, que apenas se reciben desde mediados del siglo 20 , con el resultado ya expuesto.

A lo largo de los siglos, la población local sigue sufriendo las consecuencias de una situación basada en políticas coloniales de exploración del territorio. Eduardo Galeano (1975) comentaba siempre que América Latina tiene la desdicha de las enormes riquezas que contiene, nunca siendo aprovechada por sus naturales, "el campo irradia pobreza para muchos y riqueza para muy pocos." (p. 166).

\section{EL ANÁLISIS DE LOS DISCURSOS DE LOS COMENTARIOS EN FACEBOOK MEDIANTE ALGORITMOS}

Para este trabajo desarrollamos un análisis de los comentarios extraídos de las redes sociales, en este caso, las respuestas que originó un post de Unicef Colombia.

Los análisis de redes sociales arrancan desde el mismo momento que estas empiezan a prosperar en el Internet. El trabajo de Fresno García, Daly e Sánchez-Cabezudo (2016) nos muestra que en verdad las redes son los nuevos mecanismos de influencia sobre la población siendo que su análisis es fundamental para conocer los intereses de los usuarios, por lo que se hace necesario determinar cómo se perciben los mensajes sobre un determinado tema en las redes para tener una visión del punto de vista de una población concreta.

Para Da Fonseca, Silva e Teixeira Filho (2017), las redes sociales a través del ciberactivismo son fundamentales para el proceso del empoderamiento y transformación social (p. 81) Son un mecanismo con el cual podemos percibir el impacto de acciones concretas sobre la sociedad mediante el estudio de las redes sociales.

Los trabajos de Dijk (2000) en "El estudio del discurso. El discurso como estructura y proceso" son extremadamente útiles para trabajar esta línea de investigación. Dijk (2002), nos habla del "uso actual de la lengua por usuarios concretos en situaciones sociales concretas." Esta es una interesante opción para aplicar el Análisis crítico del discurso o CDA, en un uso interdisciplinar del estudio del discurso, teniendo en cuenta cualquier manifestación lingüística, como sería el caso del estudio de los comentarios de Facebook. Se pueden observar las respuestas sociales frente a un evento concreto y el uso del lenguaje que se realiza.

El trabajo de Arrieta Bettín e Avendaño de Barón (2018) sobre el análisis linguístico, socio-discursivo y socio-pragmático en los discursos de los tweets, nos sirven para valorar esa opción para analizar una muestra representativa con el fin de obtener pautas de comportamiento frente a un evento. Tanto los mensajes de Twitter como de Facebook, u otras redes, nos permiten obtener una muestra representativa sobre un evento y analizar la misma para medir y cuantificar la respuesta de este. Según Busón (2020) los estudios sobre los comentarios nos dicen 
mucho sobre el pensamiento colectivo de un grupo social, nos revelan sus pautas de respuesta frente a una publicación concreta.

El análisis de los comentarios en Facebook nos permite observar las pautas del pensamiento de los usuarios frente a un evento concreto. En este caso, la percepción de la falta de agua en La Guajira. Los usuarios comentan lo que les parece oportuno acerca de una noticia concreta publicada en la red social, en una especie de encuesta con una pregunta abierta en un cuestionario público donde expresan lo que les dice dicha información. Dichas respuestas nos permiten realizar infinitud de estudios con estas muestras utilizando diversos algoritmos.

Entre las diversas herramientas existentes para este tipo de tratamiento de datos, nos decantamos por utilizar una herramienta basada en software libre el IRaMuTeQ (Interface de $R$ pour les Analyses Multidimensionnelles de Textes et de Questionnaires), que utiliza técnicas de análisis semántico basado en $\mathrm{R}$ como motor para los cálculos estadísticos. Desarrollado por Pierre Ratinaud (1993) que cuenta actualmente con varias traducciones y diccionarios de diversas lenguas entre ellas el español.

A través de los algoritmos de esta herramienta pudimos analizar una muestra de datos para analizar el sentir general ante un evento ya citado anteriormente. Diversos trabajos previos (RATINAUD, 2014; RATINAUD, 2016; SARRICA et al., 2016; BAVIERA, 2017 ) nos muestran las muchas posibilidades en el uso de IRaMuTeQ en análisis textual para tratar datos cualitativos.

\section{METODOLOGÍA}

\section{Herramientas utilizadas}

Para tratar esta información hemos utilizado la aplicación IRaMuTeQ versión 0.7 alfa 2 . Esta herramienta utiliza el entorno estadístico de $\mathrm{R}$, que es un entorno para cálculos estadísticos y gráficos. Como ya hemos comentado anteriormente. Mediante diversos algoritmos desarrolla varios tipos de análisis estadístico sobre los datos cualitativos de lo textos analizados, para ello es necesario transformar los datos obtenidos en un corpus de texto que posteriormente es tratado por el software. Así mismo, hicimos uso de la hoja cálculo de LibreOficce versión 5 y el Notepad ++ para tratar el corpus textual al formato requerido antes de ser procesado.

\section{Obtención de la información}

La información obtenida para este trabajo lo obtuvimos de un post de Unicef Colombia ${ }^{6}$ publicado el 15 de julio de 2020. "Con el apoyo de Unión Europea en Colombia, Claro Colombia y ZOA, entregamos kits de higiene a 60 familias de la comunidad rural \#Wayúu de Sararao, en respuesta a la emergencia por \#COVID19.". Este evento se realizó como una acción concreta para de alguna forma aliviar las duras condiciones de las comunidades Wayuu en pleno periodo de la pandemia del Covid-19. En líneas generales, fue una noticia bien recibida (figura 2) donde más del $98 \%$ la veía de forma muy positiva. Asimismo, fue compartida más de 2.200 veces lo que da una buena imagen de que era una publicación relevante entre los usuarios.

\footnotetext{
${ }^{6}$ Unicef Colombia July 15 at 7:00 PM. Con el apoyo de Unión Europea en Colombia, Claro Colombia y ZOA, entregamos kits de higiene a 60 familias de la comunidad rural \#Wayúu de Sararao, en respuesta a la emergencia por \#COVID19. Estas familias se beneficiarán de Pílas Públicas de \#AguaPotable de \#GuajiraAzul. \#UNICEFenAcción https://www.facebook.com/45235597147/posts/10158604050007148/
} 
Figura 2 - Reacciones de los usuarios ante el post en Facebook

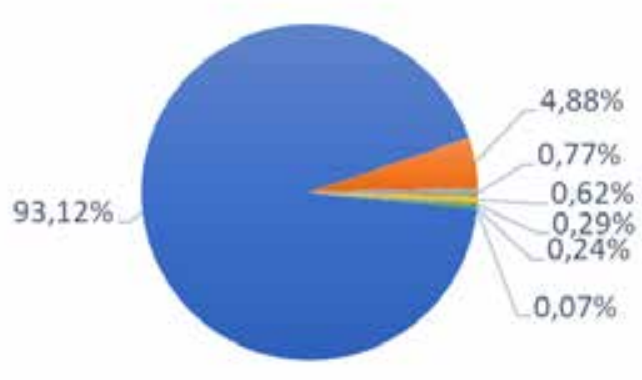

$$
\begin{aligned}
& \text { - Me gusta } \\
& \text { = Me Encanta } \\
& \text { = Me importa } \\
& \text { = Me entristece } \\
& \text { = Me sorprende } \\
& \text { - Me Divierte } \\
& \text { - Me enoja }
\end{aligned}
$$

Fuente: Facebook y elaboración propia.

Fueron obtenidos 495 comentarios válidos alojados en Facebook. Mediante una extracción de estos usando la técnica de web scraping ${ }^{7}$, a través de la cual pudimos obtener todos los comentarios en formato $\mathrm{CSV}^{8}$ que posteriormente fueron tratados para construir el corpus a analizar (tabla I).

Tabla 1 - Datos de la muestra analizada en IRaMuTeQ y R

\begin{tabular}{|l|c|}
\hline Datos & Valores \\
\hline Número de textos & $\mathrm{n}=495$ \\
\hline Número de segmentos de texto & 505 \\
\hline Número de formas & 1648 \\
\hline Número de ocurrencias & 49015 \\
\hline Número de hápax & 1853 (3.78\%de ocurrencias - 49,23 \% de formas) \\
\hline Media de ocurrencias por texto & 6163 \\
\hline Número de lemas & 1266 \\
\hline Número de formas activas & 1165 \\
\hline Número de formas suplementarias & 98 \\
\hline Número de formas activas con frecuencia & $>=3: 237$ \\
\hline Media de formas por segmento & 12,203960 \\
\hline Número de classes & 3 \\
\hline Segmentos clasificados & 397 segmentos clasificados de 505 (78.61\%) \\
\hline
\end{tabular}

Fuente: Facebook y elaboración propia mediante IRaMuTeQ y R.

\section{RESULTADOS E DISCUSIÓN}

A continuación, describiremos algunos de los resultados del análisis de los comentarios del post analizado.

\footnotetext{
${ }_{7}^{7}$ Web scraping o raspado web, es una técnica comúnmente utilizada de obtención de datos mediante la utilización de un software específico para extraer todo tipo de información de sitios web.

${ }^{8}$ CSV (del inglés comma-separated values) lo que en español sería "valores separados por comas".
} 
Análisis de los comentarios del post de Unicef Colombia del 15 de julio de 2020.

Uno de los métodos de análisis utilizado en este trabajo fue la nube de palabras, que agrupa las palabras únicamente en función de la frecuencia con que aparecen en el texto, es un método bastante sencillo y visual ya que permite observar rápidamente el peso de ciertas palabras frente a otras, para apreciar su importancia.

A continuación, realizamos los análisis de similitud, está es una técnica fundamentada en la teoría de los grafos, el cual permite la identificación de las ocurrencias de las palabras en un texto. Es otro método muy gráfico que ayuda a entender de qué forma las palabras están conectas entre sí. El resultado gráfico presentado son las indicaciones de conexión entre las mismas. Lo que nos permite identificar las ocurrencias de las palabras, su importancia en el texto y la forma en que estas se conectan.

Del mismo modo, realizamos la Clasificación jerárquico descendiente (CDH), propuesto por el método de Reinert (1993), en que los segmentos de texto (fragmentos de los textos) son clasificados de acuerdo con sus respectivos vocabularios y sus conjuntos repartidos en formas más reducidas. Es un método que nos da una clasificación de las palabras por sus classes, es decir, cómo estas están agrupadas.

Finalmente realizamos el Análisis Factorial de correspondencia (AFC), que se hace a partir de los datos obtenidos en la CHD del corpus textual. Nos permite realizar un gráfico con ejes propuestos por el modelo de Reinert, en que los segmentos de texto son clasificados de acuerdo con sus respectivos vocabularios y sus conjuntos distribuidos en formas más reducidas.

En esta nube (figura 3) nos encontramos entre las 10 primeras palabras que más se repiten en los comentarios analizados las siguientes: Dios (138 veces), gracia (73 veces), bendecir (70 veces), ayudar (58 veces), agua (48 veces), bueno (47 veces) e necesitar (39 veces). Asimismo, se observan palabras alrededor como persona, bendición, amén, guajiro, familia, comunidad, etc. Básicamente nos demuestra un agradecimiento por la ayuda prestada ante la grave situación. Sin embargo se observa que también piden una solución sostenible ante los problemas que la falta de agua provoca en el territorio.

Figura 3 - Nube de palabras del corpus analizado

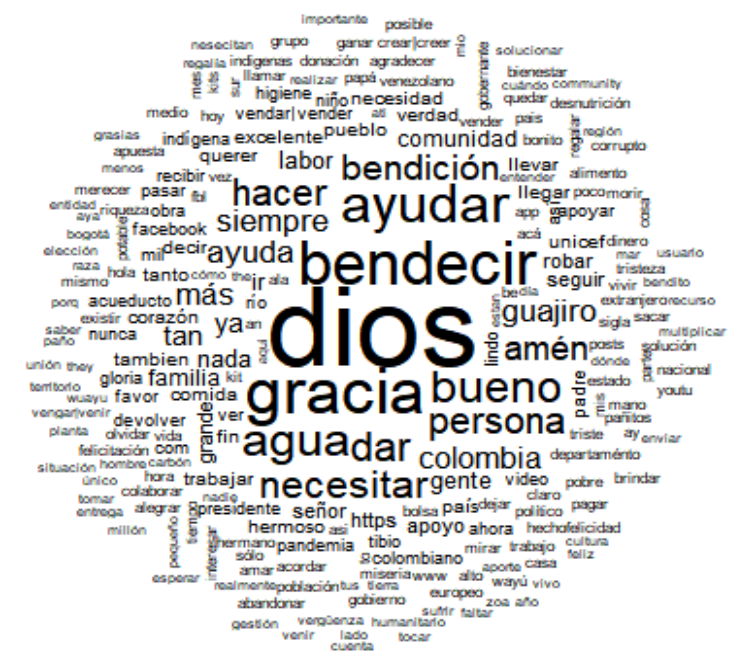

Fuente: Facebook y elaboración propia mediante IRaMuTeQ, y R. 
Figura 4 - Análisis de similitud de las palabras del corpus analizado

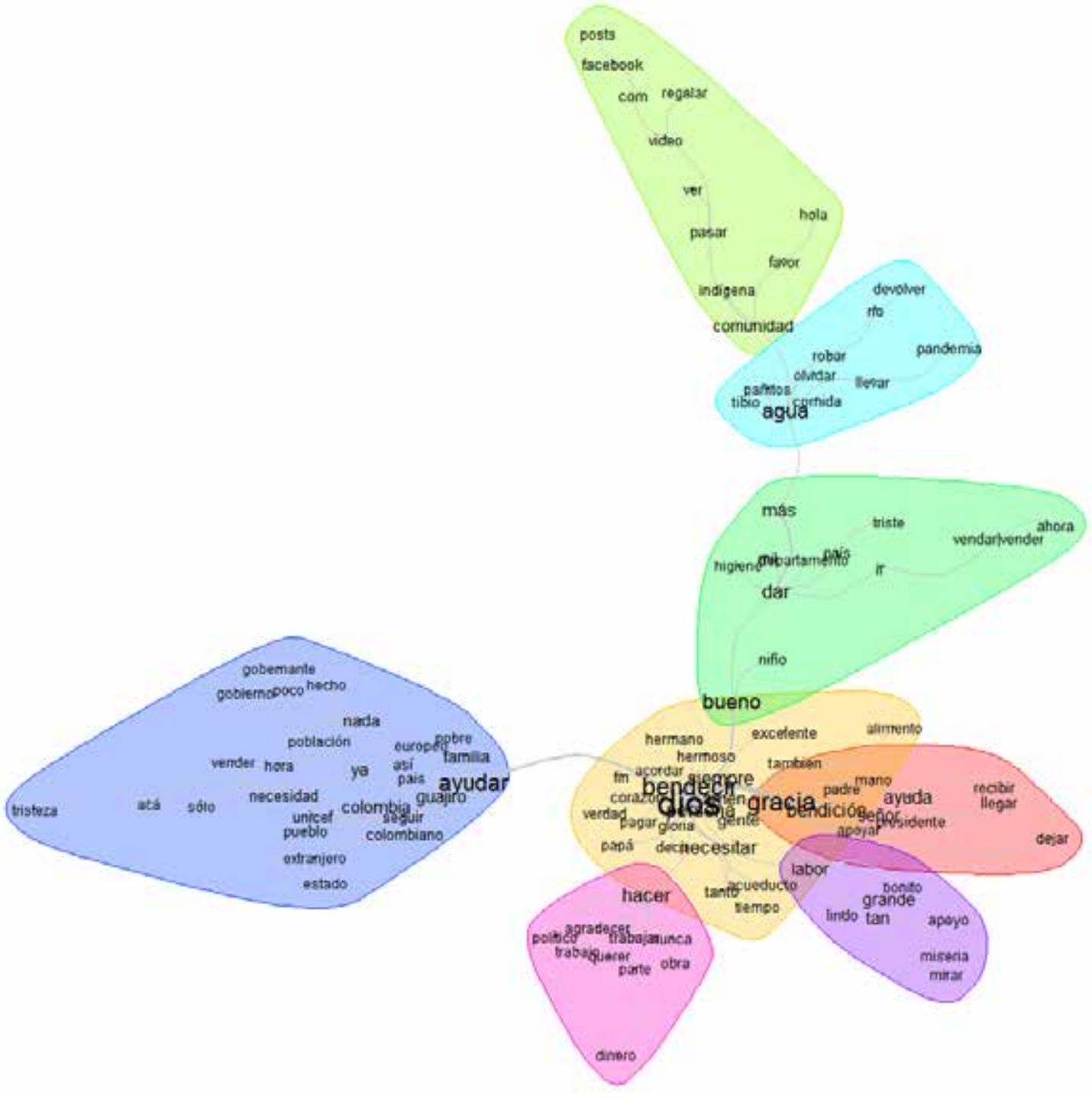

Fuente: Facebook y elaboración propia mediante IRaMuTeQ, y R.

El análisis de similitud realizado (figura 4) lo hemos representado con los halos para destacar los grupos de palabras conectadas y la relación que existe entre ellas, así como las diversas dependencias de las palabras que existen entre sí. Esta nos permite encontrar un gran grupo de palabras conectadas entre sí. Se identifica claramente que predomina un agradecimiento y sobre todo una súplica a Dios para ayudar a resolver los problemas del territorio. Adicionalmente, se puede observar en la figura lo relacionado que se encuentran el agua y las comunidades indígenas. 
Figura 5 - Dendograma de las tres clases lexicales obtenidas de la clasificación jerárquica descendente de las palabras del corpus analizado
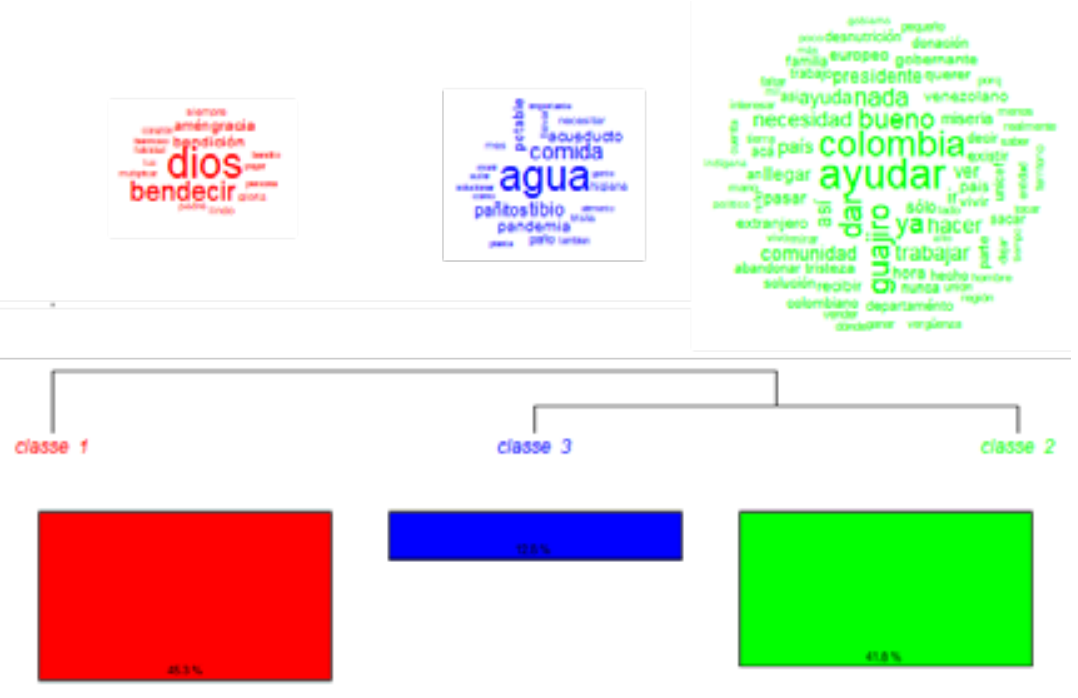

$$
\begin{aligned}
& \text { dios } \\
& \text { bendecir } \\
& \text { bendición } \\
& \text { gracia } \\
& \text { amén } \\
& \text { siempre } \\
& \text { padre } \\
& \text { lindo } \\
& \text { gloria } \\
& \text { corazón } \\
& \text { pagar } \\
& \text { persona } \\
& \text { hermoso } \\
& \text { felicidad } \\
& \text { tus } \\
& \text { multiplicar } \\
& \text { bendito } \\
& \text { claro } \\
& \text { labor } \\
& \text { excelente } \\
& \text { hoy } \\
& \text { alegrar } \\
& \text { seguir } \\
& \text { apoyo } \\
& \text { sefior } \\
& \text { raza } \\
& \text { humanitario } \\
& \text { grasias }
\end{aligned}
$$
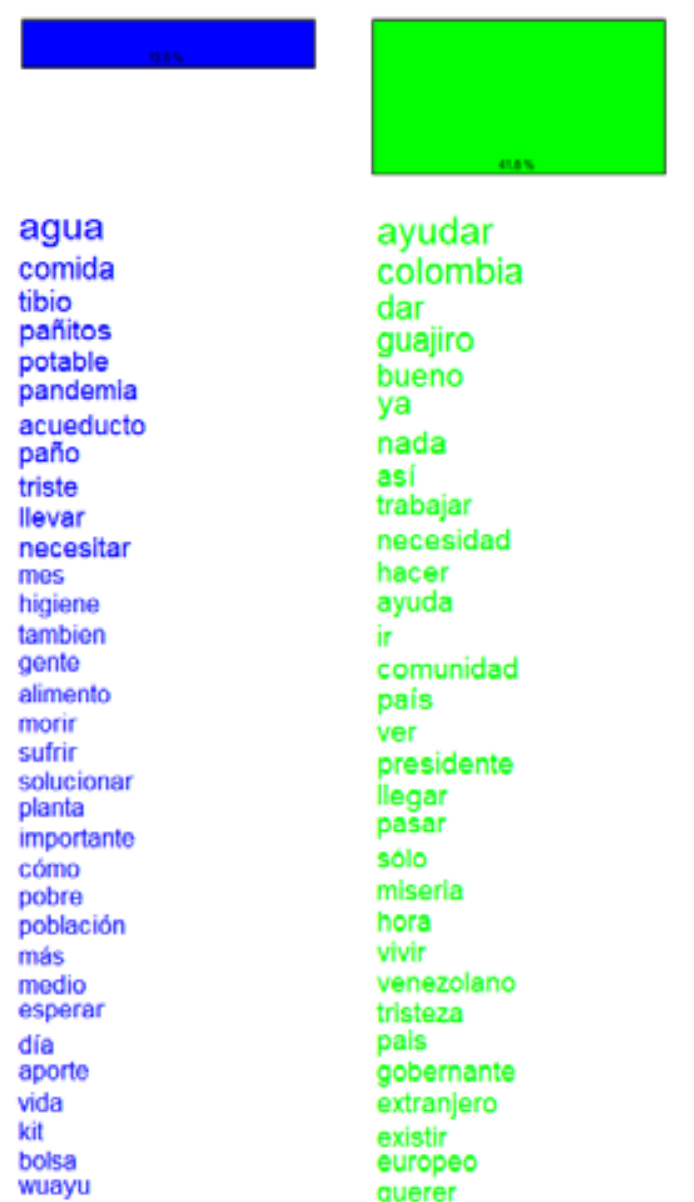

ayudar

colombia

dar

guajiro

bueno

ya

nada

así

trabajar

necesidad

hacer

ayuda

ir

comunidad

pais

ver

presidente

llegar

pasar

solo

miseria

hora

vivir

venezolano

tristeza

pals

gobernante

extranjero

existir

europeo

querer

Fuente: Facebook y elaboración propia mediante IRaMuTeQ y R.

Además, realizamos la Clasificación Jerárquica Descendente (CDH) (Figura 5) que formó un esquema jerárquico de clases de los vocabularios presentes en el corpus. Mediante el CDH pudimos obtener el contenido de cada una de las classes de los textos analizados de los discursos, en este caso fueron 3 (tres) classes; la classe 1 con un 45,3\% hace referencia a temas como Dios, bendecir, bendición, gracia, amén, etc. entre otras con un cuño eminentemente de carácter religioso. Luego, tenemos la classe 2 con un $41,8 \%$ y destacan las palabras ayudar Colombia, 
dar, guajiro, bueno, ayuda, etc. También aparece la classe 3 con un 12,8\% que hace referencia a las palabras, entre otras, a agua, comida, tibio, pañitos, pandemia, acueducto, etc.

Los comentarios indican claramente que los usuarios de la red social que comentaron, advierten la necesidad principal de acceder al agua y la denuncia de la ausencia de un sistema de distribución sostenible del agua en esta región.

Para verificarlo, extraemos unos cuantos ejemplos de los comentarios a través de la concordancia en el texto como veremos a continuación con detalle.

Tabla II - Concordancias en el texto de la palabra Guajiros a partir de las formas presentes en el corpus textual

\begin{tabular}{|c|}
\hline $\begin{array}{l}* * * * * \text { suj_0280 } \\
\text { la guajira no necesita pañitos de agua que no solucionan nada en medio del desierto la guajira necesita } \\
\text { que las regalias del carbon del gas y de la sal sean invertidas en salud educación en vías }\end{array}$ \\
\hline $\begin{array}{l}* * * * * \text { suj_435 } \\
\text { si pueden entender que la solución ni está en dar pequeñas ayudas las ayudas ni ayudan lo que mis } \\
\text { guajiros necesitan es que le devuelvan su río que las regalías del carbón sean invertidas en universida- } \\
\text { des hospitales }\end{array}$ \\
\hline $\begin{array}{l}* * * * * \text { suj_0327 } \\
\text { no hubo otro medio donde darles las donaciones a los guajiros la guajira no aguanta mas la contamina- } \\
\text { ción con bolsas plásticas }\end{array}$ \\
\hline $\begin{array}{l}* * * * * \text { suj_0344 } \\
\text { paños de agua tibia y no solucionan los verdaderos problemas de la guajira el hambre y la sed cómo si } \\
\text { fueran a comer antibacteriales y qué los niños se nutran con jabón }\end{array}$ \\
\hline $\begin{array}{l}\text { *****suj_0097 } \\
\text { más bien porq no ven lo q realmente pasa en la guajira q hay tanta desnutrición y miseria le } \\
\text { borar con algo tan básico guajira es tierra sin len no hay ningun mandatario q luche por esa }\end{array}$ \\
\hline
\end{tabular}

Fuente: Facebook y elaboración propia mediante IRaMuTeQ y R.

Tabla III - Concordancias en el texto de la palabra agua a partir de las formas presentes en el corpus textual

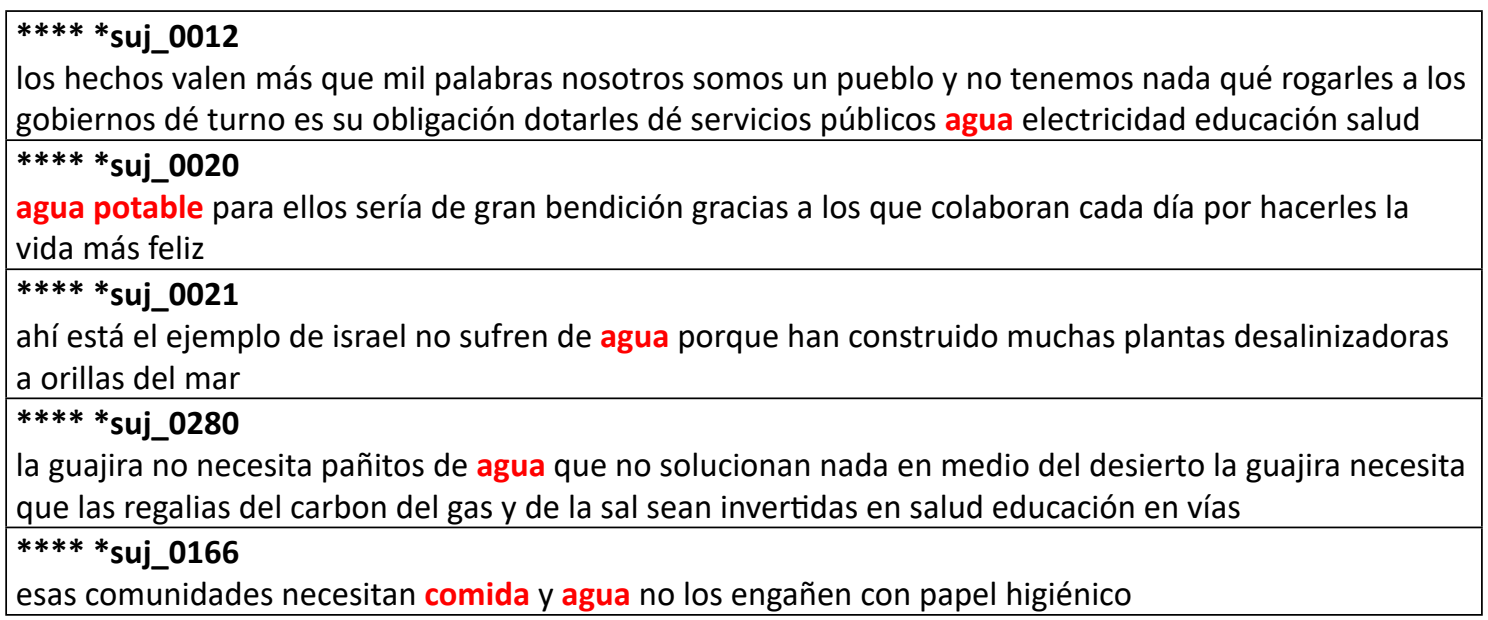
Fuente: Facebook y elaboración propia mediante IRaMuTeQ y R.

Observando las concordancias (Tabla II y Tabla III) extraídas de la muestra podemos percibir una crítica y al mismo tiempo un agradecimiento ante la difícil situación del territorio. La 
ciudadanía percibe de forma clara la gravedad de la situación, aunque, también exige soluciones permanentes.

Figura 6 - Análisis de Especificidades y AFC de las tres classes lexicales de las palabras del corpus

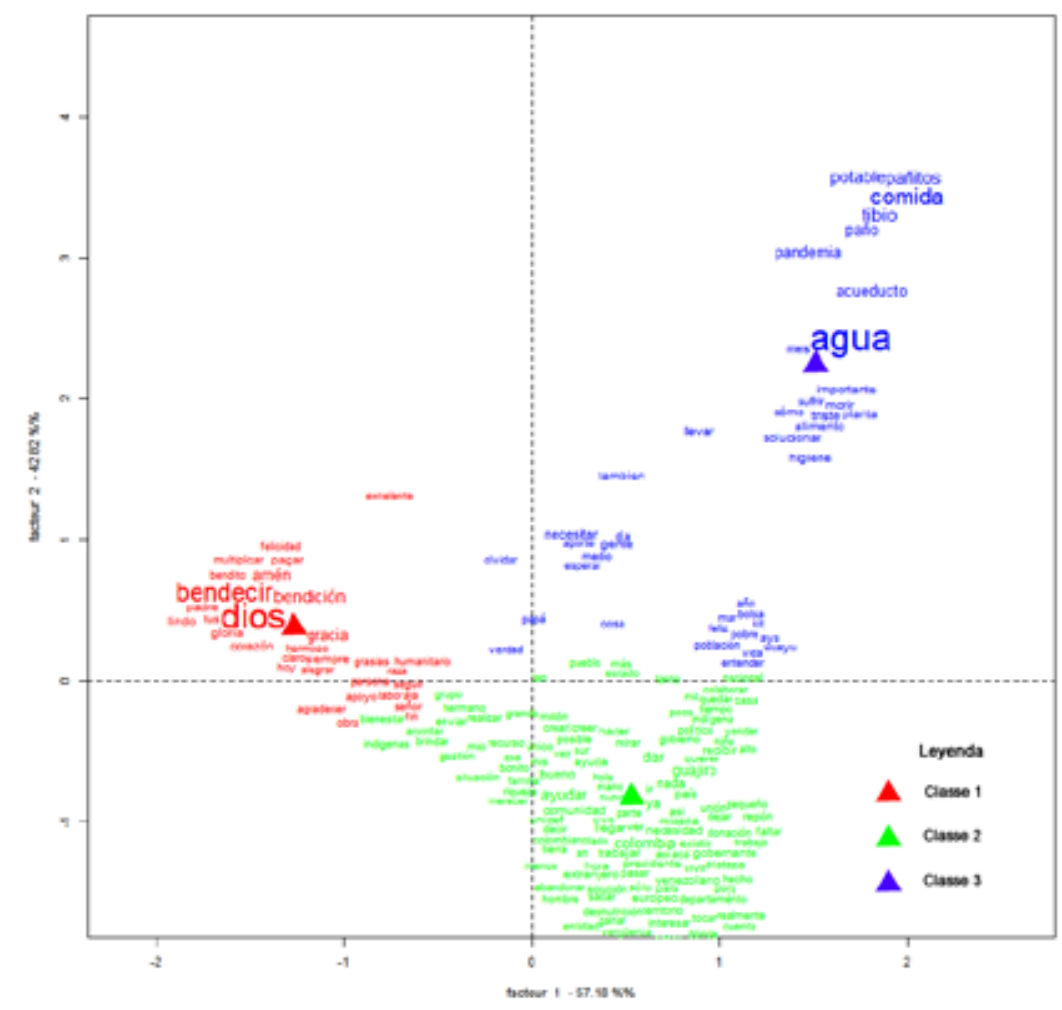

Fuente: Facebook y elaboración propia mediante Itamuteq y R.

Del análisis del AFC (figura 6) resultaron tres classes, el eje $X$ está representado el factor 1 en un $57,18 \%$ y el Y con un factor de $42,82 \%$ del cuerpo textual. La distribución de clases quedó representada de la siguiente forma: la classe 1 quedó localizada en el cuadrante del eje negativo $X \mathrm{Y}$ el positivo $Y$, se encuentra en el cuadrante superior izquierdo. La classe 2 quedó restringida a la parte positiva de X, no obstante, está localizada en la parte negativa del eje Y. Mientras que la classe 3 se encuentra en la parte positiva de $X$ y del eje $Y$. Las classes 2 y 3 estas relacionadas entre sí, mientras que la classe 1 corresponde a otro grupo de palabras poco relacionadas con las anteriores.

\section{CONCLUSIONES}

En este trabajo pudimos apreciar las posibilidades de analizar a través de técnicas de textometría los comentarios existentes en un post de Facebook y de otras redes sociales, esta vez nos permitió observar cómo es percibido un problema concreto por parte de la población. Pese a las limitaciones descritas por Chartier e Meunier (2011) donde aseguran que la fascinación por la tecnología puede mermar los objetivos reales de la investigación, por eso es importante el papel del investigador en el uso de este tipo de análisis.

Esta pequeña investigación hace parte de un proyecto más amplio para estudiar la valoración de ecosistemas costeros en relación con las prácticas culturales de la comunidad Wayuu de Mayapo, La Guajira, Colombia, en la Maestría en Ciencias Sociales de la Universidad de La Guajira. 
LA PERCEPCIÓN DE LA FALTA DE AGUA EN LAS COMUNIDADES WAYUU EN LA GUAJIRA (COLOMBIA). UNA PROPUESTA PARA EL USO DE LAS REDES SOCIALES COMO HERRAMIENTA DE ANÁLISIS DE DESARROLLO

Katherin Pérez Mendoza - Carlos Busón Buesa

Pudimos comprobar que dicha herramienta es bastante útil para procesar grandes volúmenes de datos que envuelvan textos, como pueden ser los comentarios de las redes sociales. El análisis estadístico nos ayudó a visualizar la percepción sobre un determinado post, pudiendo obtener datos cualitativos y cuantitativos; lo que, sin duda, posibilita al investigador a disponer de una interesante herramienta para el análisis de información textual con profundidad. El software permite realizar numerosas simulaciones que ayudan al investigador a poder realizar investigaciones más específicas sobre puntos que destaquen la atención.

En cuanto a las respuestas, estas indican que el problema requiere soluciones que son conocidas por la población, como la disponibilidad de agua y la ausencia de infraestructuras para llevar la misma a las comunidades vulnerables.

\section{REFERENCIAS}

ARRIETA-BETTIN, L. E.; AVENDANO-DE BARON, G. S. El discurso del tuit: un análisis lingüístico, sociodiscursivo y sociopragmático. Cuad. linguist. hisp., Boyacá, n. 32, p. 107-130, 2018.

BAVIERA, T. Técnicas para el análisis del sentimiento en Twitter: Aprendizaje Automático Supervisado y SentiStrength. Dígitos, 1(3), 33-50. 2017

BAYONA VELASQUEZ, E. M.; Producción de carbón y crecimiento económico en la región minera del Caribe colombiano. Rev. Econ. Caribe, Barranquilla, n. 17, p. 1-38, 2016. https://doi.org/10.14482/ecoca.17.8452.

BONET-MORÓN, J. A.; HAHN-DE-CASTRO, L. W.; HAHN-DE-CASTRO, L. W. La mortalidad y desnutrición infantil en La Guajira. Documentos de Trabajo Sobre Economía Regional y Urbana, n. 255, 2017.

BOUCHET-VALAT, M.; BASTIN, G. RcmdrPlugin. Temis, a graphical integrated text mining solution in R. The $R$ Journal, 5(1), p. 188-196, 2013.

BUSÓN, C. La minería de opinión para el análisis del discurso de odio en las redes sociales. Opinion mining for the analysis of hate speech in social networks: Un estudio de caso sobre Paulo Freire en YouTube durante el periodo 2007-2019. (A case study on Paulo Freire on YouTube during the period 2007-2019). Commons. Revista de Comunicación y Ciudadanía Digital, 9(1), p. 119-159, 2020. Disponível em: https:// revistas.uca.es/index.php/cayp/article/view/5482

CHARTIER, J. F.; MEUNIER, J. G. Text mining methods for social representation analysis in Large Corpora. Papers on Social Representations, 20(37), 1-47, 2011.

CURIEL, E. B. M. Malnutrition in children of the Wayuu ethnicity: among the ethical, the unique and the relevant. Revista Médica Electrónica, 39(S1), p. 803-812, 2017.

DA FONSECA, S. M. M.; SILVA, A. P. da; TEIXEIRA FILHO, J. G. de A. O impacto do ciberativismo no processo de empoderamento: o uso de redes sociais e o exercício da cidadania. In: Desenvolvimento em Questão, v. 15, n. 41, p. 59-84, 2017. DOI: 10.21527/2237-6453.2017.41.59-84. Disponível em: https://www.revistas. unijui.edu.br/index.php/desenvolvimentoemquestao/article/view/4375. Acesso em: 15 mayo 2021.

DANE. Censo Nacional de Población y Vivienda - CNPV - 2018. Dirección de Censos y Demografía - DCD/ Departamento Administrativo Nacional de Estadística, 2019.

DAZA CALDERÓN, N. A.; CORREDOR MORA, D. M. Estudio del grado de vulnerabilidad por escasez de agua potable en tres municipios de La Guajira, 2016. Disponível em: https://ciencia.lasalle.edu.co/ing_civil/64

DAZA-DAZA, A. R.; RODRÍGUEZ-VALENCIA, N.; CARABALÍ-ANGOLA, A. El Recurso Agua en las Comunidades Indígenas Wayuu de La Guajira Colombiana. Parte 1: Una Mirada desde los Saberes y Prácticas Ancestrales. Información Tecnológica, 29(6), p. 13-24, 2018.

DEL FRESNO GARCÍA, M.; DALY, A. J.; SÁNCHEZ-CABEZUDO, S. S. Identificando a los nuevos influyentes en tiempos de Internet: medios sociales y análisis de redes sociales (identifying the new influencers in the Internet Era: social media and social network analysis). Reis: Revista Española de Investigaciones Sociológicas, p. 23-42, 2016.

DIJK, T. A. V. El análisis crítico del discurso y el pensamiento social. Athenea Digital. Revista de Pensamiento e Investigación Social, 1(1), p. 18-24, 2002. Disponível em: https://doi.org/10.5565/rev/athenead/ v1n1.22 
LA PERCEPCIÓN DE LA FALTA DE AGUA EN LAS COMUNIDADES WAYUU EN LA GUAJIRA (COLOMBIA). UNA PROPUESTA PARA EL USO DE LAS REDES SOCIALES COMO HERRAMIENTA DE ANÁLISIS DE DESARROLLO

Katherin Pérez Mendoza - Carlos Busón Buesa

DIJK, T. A. V. El estudio del discurso. In: DIJK, T. A. V. El discurso como estructura y proceso. Barcelona: Gedisa. 2000. p. 21-65.

FAÇANHA, I. P. Gênero e água: uma leitura sobre as políticas no semiárido e a inclusão feminina. In: Desenvolvimento em Questão, v. 17, n. 47, p. 339-356, 2019. DOI: 10.21527/2237-6453.2019.47.339-356. Disponível em: https://www.revistas.unijui.edu.br/index.php/desenvolvimentoemquestao/article/ view/7536. Acesso em: 15 mayo 2021.

GALEANO, E. Las venas abiertas. América Latina. Buenos Aires: Siglo, 1975.

JABBA, A. S. "El gas de La Guajira y sus efectos económicos sobre el departamento." (Serie Documentos de Trabajo Sobre Economía Regional). Cartagena: Banco de la República. ISSN 1692/3715, 2011.

JUSTO, A. M.; CAMARGO, B. V. Estudos qualitativos e o uso de softwares para análises lexicais. 2014. In: NOVIKOFF, C.; SANTOS, S. R. M.; MITHIDIERI, O. B. (org.). Caderno de artigos: X SIAT \& II Serpro. Duque de Caxias: Universidade do Grande Rio "Professor José de Souza Herdy" - Unigranrio, 2014. p. 37-54. Disponível em: https://lageres.wordpress.com/

LAUER, S. A. et al. The Incubation Period of Coronavirus Disease 2019 (COVID-19) From Publicly Reported Confirmed Cases: Estimation and Application. Ann Intern Med., 2020. [Epub ahead of print 10 March 2020]. DOI: https://doi.org/10.7326/M20-0504

LEÓN LINARES, E.; ACOSTA ARIAS, C. M. Análisis de vulnerabilidad del territorio por sequía en el departamento de La Guajira, Colombia, a partir de una visión basada en necesidades básicas insatisfechas. 2015. Bachelor's (Thesis) - Universidad Católica de Colombia Facultad de Ingeniería Programa de Ingeniería Civil, Bogotá, 2015.

MCALOON, C. G. The incubation period of COVID-19 - A rapid systematic review and meta-analysis of observational research. medRxiv preprint. 2020. DOI: https://doi.org/10.1101/2020.04.24.20073957. Disponível em: 5 mar. 2021.

MEISEL-ROCA, A. La Guajira y el mito de las regalías redentoras. Documentos de Trabajo Sobre Economía Regional y Urbana, n. 86, 2007.

MENDOZA, K. P. Hilos por Mma. Una propuesta de economía circular a través de la educación ambiental en La Guajira, Colombia. Encontro Internacional de Gestão, Desenvolvimento e Inovação (Eigedin), v. 4, n. 1, 2020.

MORÓN, J. A. B.; DE CASTRO, L. W. H. La mortalidad y desnutrición infantil en La Guajira. Revista del Banco de la República, 90(1.074), p. 29-58, 2017.

OSORIO, A. et al. Influencia del Contexto Socioeconómico de la Comunidad sobre la Desnutrición Infantil en Colombia: un Enfoque Multinivel Para Los Años 2005 y 2010 (Influence of Community Socioeconomic Context on Child Malnutrition in Colombia: A Multilevel Approach for 2005 and 2010). 2016. Disponível em: SSRN 2796162.

RATINAUD, P. Étude d'un large corpus textuel avec IRaMuTeQ: Twitter et le hashtag\# mariagepourtous. Les représentations sociales. Théories, méthodes et applications. Louvain-la-Neuve: De Boeck Supérieur, 2016. p. 107-112.

RATINAUD, P. Visualisation chronologique des analyses Alceste: application à Twitter avec l'exemple du hashtag\# mariagepourtous. Actes des 12es Journées internationales d'Analyse statistique des Données Textuelles. Paris Sorbonne Nouvelle-Inalco. 2014. Disponível em: http://lexicometrica.univ-paris3.fr/ jadt/jadt2014/01-ACTES/46-JADT2014.pdf. Acesso em: 5 mar. 2021.

RATINAUD, P. Les "mondes lexicaux" et leur "logique" à travers l'analyse statistique d'un corpus de récits de cauchemars. Langage et société, n. 66, p. 5-39, 1993.

REINERT, M. Les "Mondes lexicaux" et leur "logique" à travers de l'analyse statistique d'un corpus de récits de cauchemars. Langage et société, v. 66, n. 1, p. 5-39, 1993.

REIS, C. A. S.; CARNEIRO, R. O direito humano à água e a regulação do saneamento básico no Brasil: tarifa social e acessibilidade econômica. In: Desenvolvimento em Questão, v. 19, n. 54, p. 123-142, 2021. DOI: 10.21527/2237-6453.2021.54.123-142. Disponível em: https://www.revistas.unijui.edu.br/index.php/desenvolvimentoemquestao/article/view/10995. Acesso em: 15 mayo 2021.

RUSSELL, E. A. et al. Childhood malnutrition within the indigenous Wayuú children of northern Colombia. Global Public Health, 15(6), p. 905-917, 2020.

SARRICA, M. et al. The effects of lemmatization on textual analysis conducted with IRaMuTeQ: results in comparison. 13ème Journées internationales d'Analyse statistique des Données Textuelles. 2016. Disponível em: http://lexicometrica.univ-paris3.fr/jadt/jadt2016/01-ACTES/82897/82897.pdf. Acesso em: 12 mar. 2021.

SILVA ORTEGA, J.; OJEDA CAMARGO, E.; CANDELO, J. Perspectivas de Comunidades Indígenas de La Guajira Frente al Desarrollo Sostenible y el Abastecimiento Energético. Revista Espacios, v. 38, n. 11, 2017.

SOUZA, M. et al. O uso do software IRAMUTEQ na análise de dados em pesquisas qualitativas. Revista da Escola de Enfermagem da USP, 52, 2018. Disponível em: https://10.1590/s1980-220x2017015003353. Acesso em: 5 mar. 2021. 